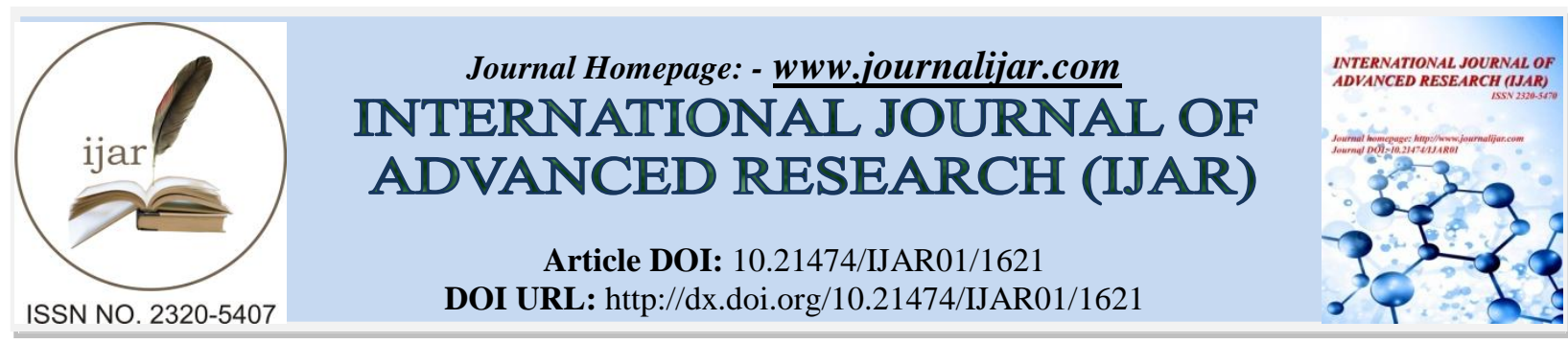

RESEARCH ARTICLE

\title{
OSTEOMETRIC ANALYSIS OF EAR OSSICLES IN POPULATION OF RAJASTHAN.
}

\author{
Shweta Asthana ${ }^{1}$, Sandhya Mehra ${ }^{1}$, Aarushi Jain ${ }^{2}$ and Kalpna Makheja ${ }^{2}$. \\ 1. Senior Demonstrator, Dept. Of anatomy, GMC Kota. \\ 2. Asst. Professor, Dept. Of anatomy, GMC Kota.
}

\section{Manuscript Info}

Manuscript History

Received: 12 July 2016

Final Accepted: 22 August 2016

Published: September 2016

Key words:-

\section{Abstract}

Background: It has been long known fact that mammalian ear ossicles have evolved from transformation of hyomandibular cartilage, quadrate and articular bone which transmits sound waves to inner ear.

Aims and objectives: This study was an attempt to analyse morphometry of three ear ossicles and to deduce a correlation between their dimensions.

Material and methods: 30 human cadavers were dissected to extract the temporal bones of both the sides and the ear ossicles were procured and all the ear ossicles were analysed for their osteometric dimensions using medcalc software.

Observation and conclusion: The distance between the tympanic part of facial nerve and the ossicles were also noted approximately $3.02 \mathrm{~mm}$ as it is an important structure to be preserved in ossiculoplasty. A positive correlation was found between the dimensions and the respective weights of the ossicles which is useful for designing the ossicular implants.

Key words: Ear ossicles, facial nerve, Osteometric analysis, middle ear, digital vernier calliper

Introduction: Middle ear bones have evolved from hyomandibular cartilage of amphibians, reptiles and birds which suspends upper jaw from skull. These transmits sound wave into inner ear. In mammals two ear ossicles malleus and incus have been derived from quadrate and articular bone which in lower land vertebrates intervene between lower jaw and skull bone [1].Thus any deformity or damage to the ossicles may lead to conductive hearing loss which may be corrected by using middle ear prosthesis. Thus this study was an attempt to analyse morphometry of the three ear ossicles and also to deduce any correlation between their dimensions so as to aid the prosthesis design and its manufacture.

Material and method:- Present study was performed in 30 human cadavers. The skull bone was confiscated and brain was removed carefully according to Cunningham's manual of practical anatomy. The tegmen tympani was chipped off by using micro motor, chisel and hammer and the three ear ossicles were 
removed and the dimensions were taken by digital vernier calliper with resolution of $0.01 \mathrm{~mm}$. Weight was taken by digital analytical balance with the sensitivity of $0.01 \mathrm{mg}$. The course of facial nerve was also observed and its distance with the ossicles was also measured with digital vernier calliper. All the results which were obtained were analysed using medcalc software. The angle of incus was measured using sketch up pro 2014 software.

Result and observation: Mean distance was calculated from head of malleus to the tympanic part of facial nerve as $3.02 \mathrm{~mm}$ (fig 1).

Copy Right, IJAR, 2016,. All rights reserved.

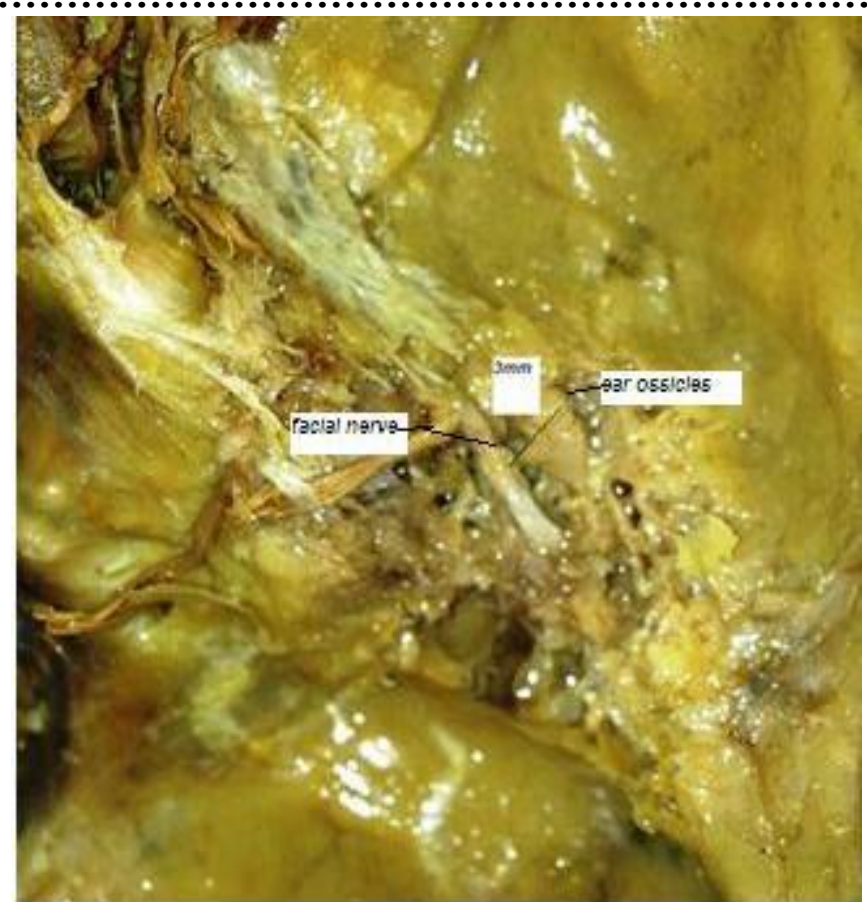

Fig 1:- (Prosection of Temporal Bone showing facial nerve and ear ossicles)

The following dimensions were measured in malleus (fig 2), incus (fig 3) and stapes (fig4,5) and recorded as per table $1,2,3,4,5$ and 6

1. Malleus

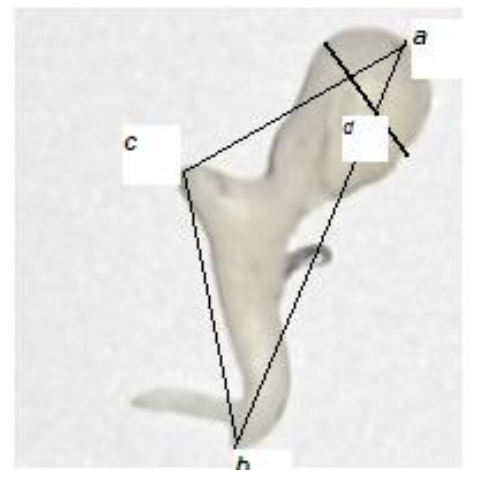

fig 2:- (malleus)

Total length: $a-b$

Length of manubrium or handle: $b-c$

Length of head and neck :( a-c) 
Results were recorded as per in table no. 1 and 2 for malleus, table no. 3 and 4 for incus and table no. 5 and 6 for stapes.

Table no.1:- (Dimensions of malleus)

\begin{tabular}{|l|l|l|l|l|}
\hline \multirow{2}{*}{ Malleus Parameters(mm) } & \multicolumn{2}{|c|}{ RT } & \multicolumn{2}{c|}{ Lt } \\
\cline { 2 - 5 } & mean & \pm Sd & mean & \pm Sd \\
\hline Total length(a-b) & 7.56 & 0.39 & 7.97 & 0.34 \\
\hline Length of handle(b-c) & 4.63 & 0.31 & 4.48 & 0.6 \\
\hline Head and Neck(a-c) & 4.54 & 0.4 & 4.51 & 0.22 \\
\hline Diameter of Head(d) & 2.54 & 0.41 & 2.34 & 0.20 \\
\hline Weight(mg) & 23.49 & 0.92 & 23.5 & 0.2 \\
\hline
\end{tabular}

(Rt -right, Lt- left)

Graph no.1:-(comparision between $\mathrm{rt}$ and lt sided malleus)

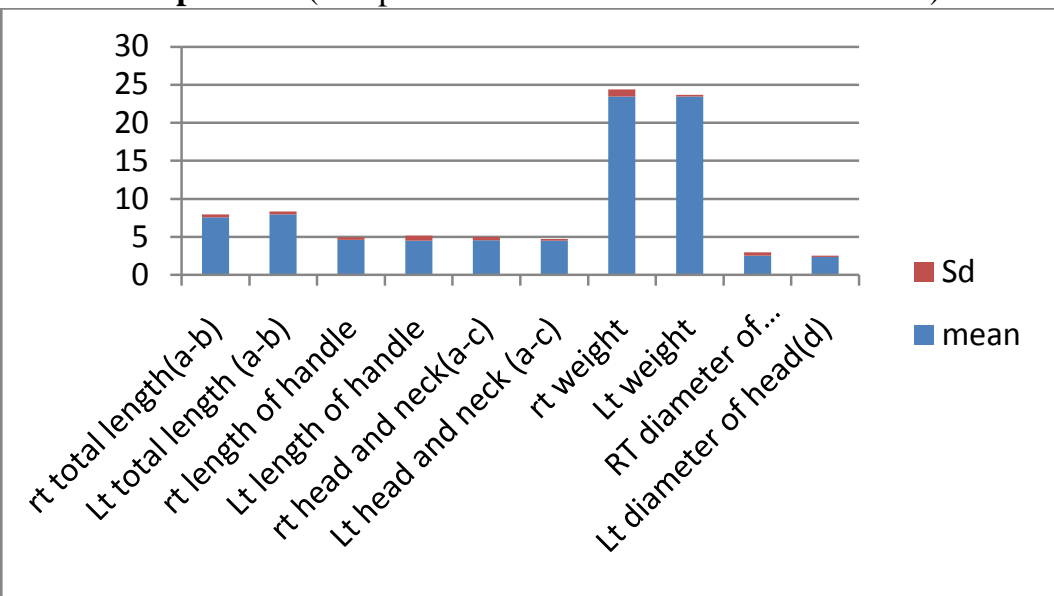

Table no. 2:- (Regression equation between various dimension of malleus and their respective weight)

\begin{tabular}{|c|c|c|c|c|}
\hline \multicolumn{2}{|l|}{ Variable } & \multirow{2}{*}{$\begin{array}{l}\text { Coeff of } \\
\text { determination }\left(\mathrm{R}^{2}\right)\end{array}$} & \multirow[t]{2}{*}{$\mathrm{P}$ value } & \multirow[t]{2}{*}{ Regression equation } \\
\hline Dependent(y) & Independent(x) & & & \\
\hline Rt malleus Weight & Rt total length(a-b) & 0.7451 & $<0.0001$ & $\mathrm{y}=12.6817+1.4115 \mathrm{x}$ \\
\hline Lt malleus Weight & Lt total length(a-b) & 0.8045 & 0.0027 & $y=-10.4265+4.4508 x$ \\
\hline Rt malleus Weight & Rt length_of_handle(b-c) & 0.2629 & $<0.0001$ & $\mathrm{y}=18.7581+1.0011 \mathrm{x}$ \\
\hline Lt malleus Weight & Lt length_of_handle(b-c) & 0.3566 & $<0.0001$ & $\mathrm{y}=17.4893+1.3400 \mathrm{x}$ \\
\hline Rt malleus Weight & Rt head and neck(a-c) & 0.5397 & $<0.0001$ & $\mathrm{y}=13.8492+2.0324 \mathrm{x}$ \\
\hline Lt malleus Weight & Lt head and neck(a-c) & 0.9404 & 0.0022 & $y=-4.4774+6.1176 x$ \\
\hline Rt malleus Weight & Rt diameter of head(d) & 0.7516 & $<0.0001$ & $y=15.2542+3.3979 x$ \\
\hline Lt malleus Weight & Lt diameter of head(d) & 0.7940 & $<0.0001$ & $y=11.3523+4.9806 x$ \\
\hline
\end{tabular}

(Rt -right ,lt left)

2.Incus:- ( fig 3):

Length of long process $\mathrm{mm}$ (d-e)

Length of short process $(\mathrm{mm})=(\mathrm{d}-\mathrm{f})$

distance between two process $=(e-f)$

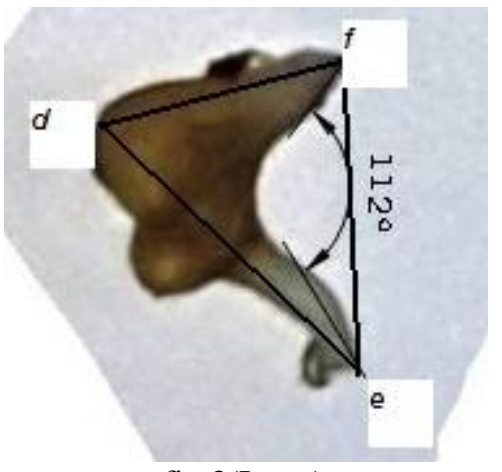

fig 3(Incus) 
Table 3:- (Dimensions of Incus)

\begin{tabular}{|l|l|l|l|l|}
\hline \multirow{2}{*}{ Incus Parameters(mm) } & \multicolumn{2}{|c|}{ RT } & \multicolumn{2}{c|}{ Lt } \\
\cline { 2 - 5 } & mean & \pm Sd & mean & \pm Sd \\
\hline Long process (de) & 6.57 & 0.45 & 6.52 & 0.44 \\
\hline Short process(df) & 4.94 & 0.34 & 5.99 & 0.4 \\
\hline Distance b/w two (ef) & 4.54 & 0.4 & 4.51 & 0.22 \\
\hline Angle $^{\circ}$ & $106.00^{\circ}$ & 3.73 & $106.67^{\circ}$ & 3.79 \\
\hline Weight(mg) & 26.62 & 1.56 & 26.94 & 1.22 \\
\hline
\end{tabular}

(Rt- right ,Lt- left, , Wt -weight)

Graph 2:-(Comparison between right and left incus)

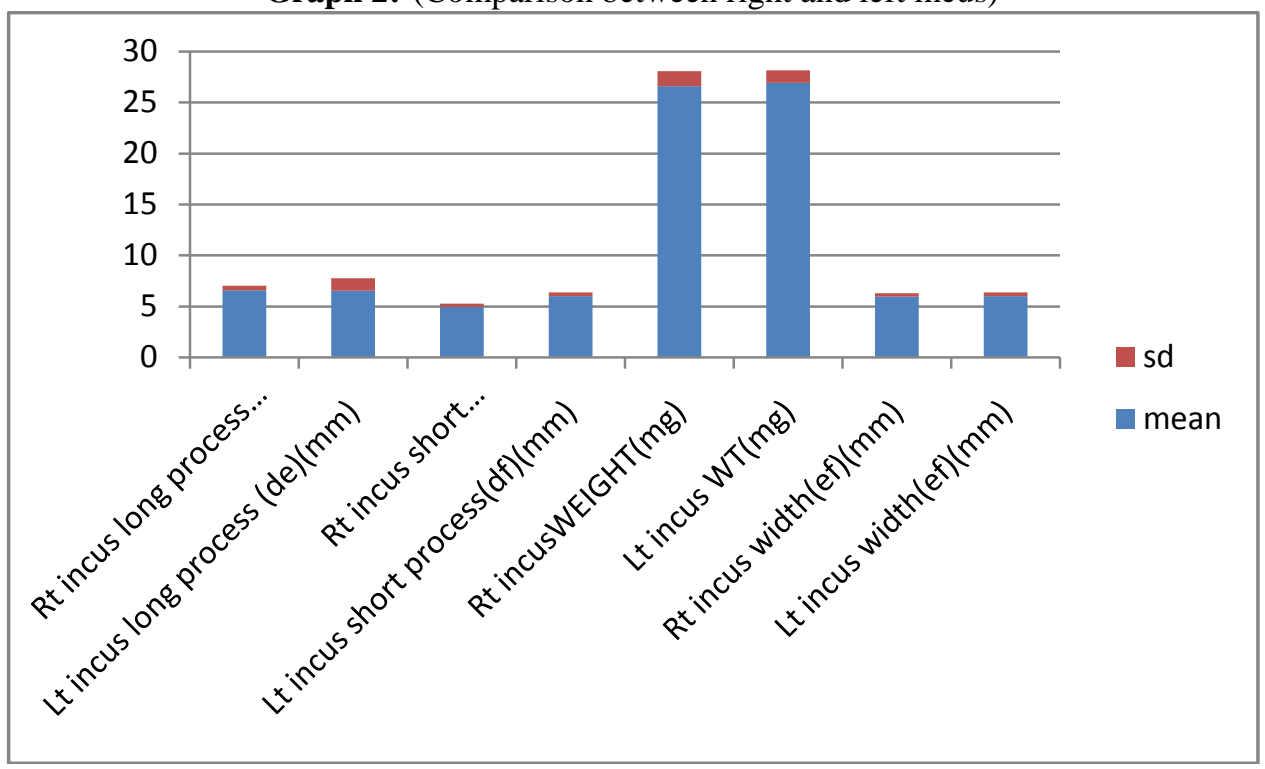

Regression coefficient with weight was also calculated as per table no.4 and Pearson correlation coefficient between width and angle of right and left incus was $\mathbf{0 . 6 6}$ and $\mathbf{0 . 8 6}$ respectively.

Table no. 4:-(Regression equation between various dimension of incus and their respective weights)

\begin{tabular}{|l|l|l|l|l|}
\hline Variable & $\begin{array}{l}\text { Coeff. Of Determination } \\
\left(\mathrm{R}^{2}\right)\end{array}$ & $\mathrm{P}$ value & Regression equation \\
\hline Dependent(y) & Independent(x) & 0.6314 & $<0.0001$ & $\mathrm{y}=9.8531+2.5523 \mathrm{x}$ \\
\hline Rt incus Weight & Rt incus long process & 0.7757 & $<0.001$ & $\mathrm{y}=11.0353+2.4370 \mathrm{x}$ \\
\hline Lt incus Weight & Lt incus long process & 0.1032 & $<0.0001$ & $\mathrm{y}=19.8064+1.3844 \mathrm{x}$ \\
\hline Rt incus Weight & Rt incus short process & 0.01 & $<0.0001$ & $\mathrm{y}=25.0598+0.3832 \mathrm{x}$ \\
\hline Lt incus Weight & Lt incus short process & 0.01 & \\
\hline
\end{tabular}

(Rt- right ,Lt- left)

Stapes:

Height $=(\mathrm{g}-\mathrm{h})$

Length of Footplate $=(i-j)$

Breadth of Footplate $=(\mathrm{k}-\mathrm{l})$
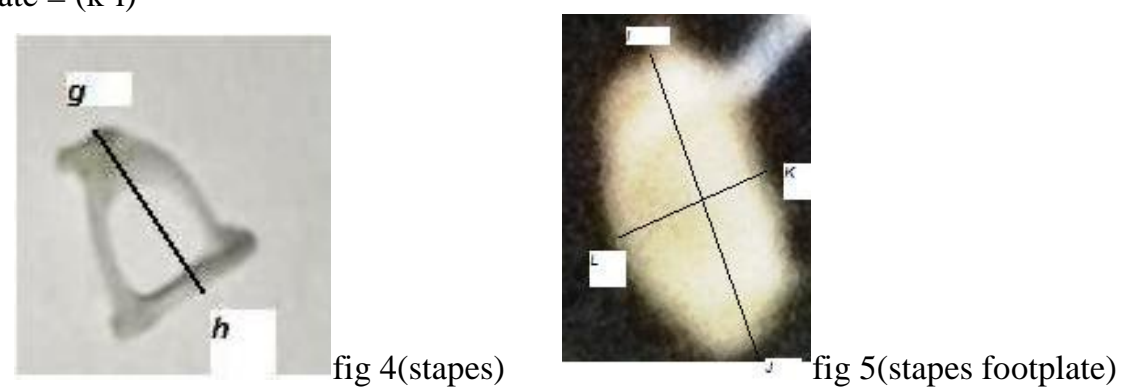
Table 5:- (Dimension of stapes)

\begin{tabular}{|l|l|l|l|l|}
\hline \multirow{2}{*}{ Stapes parameters } & \multicolumn{2}{|l|}{ Rt } & Lt & \pm sd \\
\cline { 2 - 5 } & mean & \pm sd & mean & 0.21 \\
\hline Lt OF fp(mm)(i-j) & 3.05 & 0.11 & 2.98 & 0.08 \\
\hline Breath Of fp(mm)(k-1) & 1.18 & 0.11 & 1.2 & 0.1 \\
\hline Weight(mg) & 3.35 & 0.26 & 3.2 & 0.17 \\
\hline Ht(g-h)(mm) & 3.23 & 0.08 & 3.29 & \\
\hline
\end{tabular}

(Rt- right ,Lt- left, fp- foot plate, Ht -height)

Graph 3:- Comparison between right and left stapes.

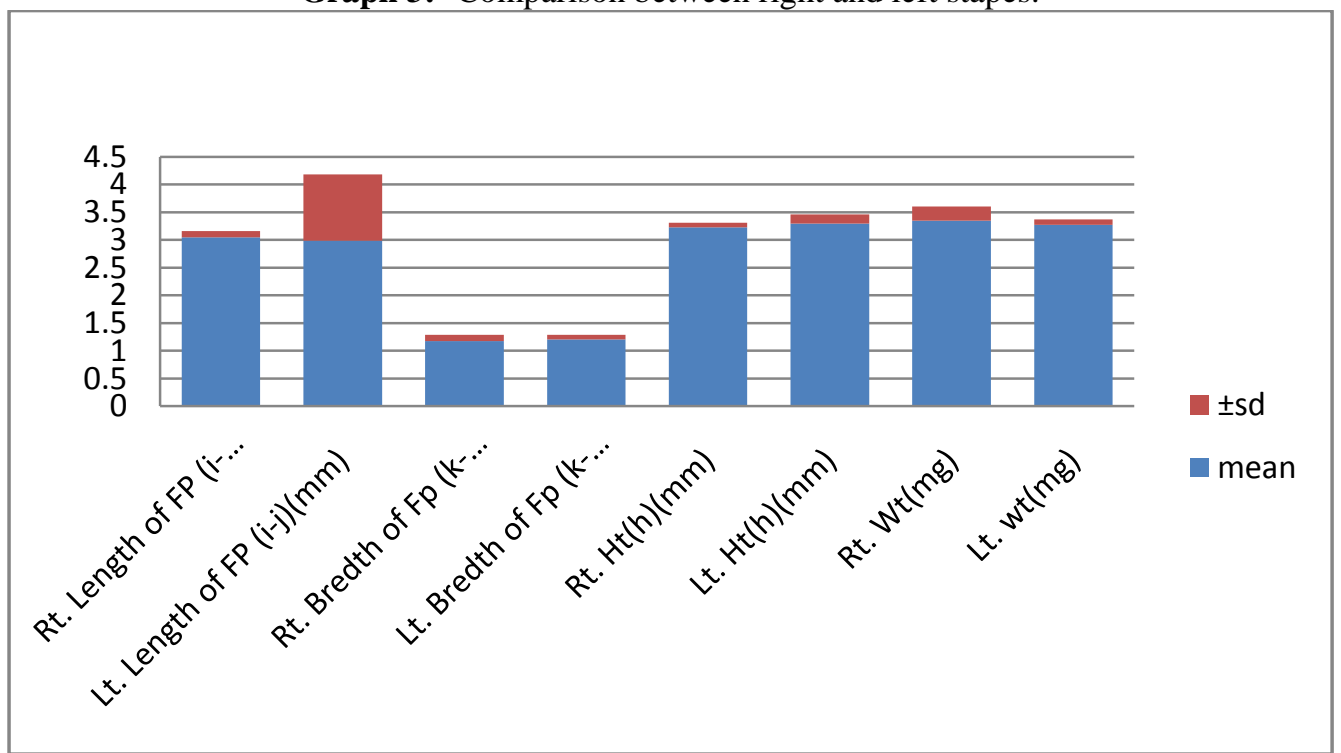

(Rt- right ,Lt- left, fp- foot plate, Ht -height)

Table 6:-(Regression equation between various dimension of incus and their respective weight)

(Rt -right,Lt -left, Coeff-coefficient)

\begin{tabular}{|c|c|c|c|c|}
\hline \multicolumn{2}{|l|}{ Variable } & \multirow{2}{*}{$\begin{array}{l}\text { Coeff. } \\
\text { Determination } \\
\left(\mathrm{R}^{2}\right)\end{array}$} & \multirow[t]{2}{*}{$\mathrm{P}$ value } & \multirow[t]{2}{*}{ Regression equation } \\
\hline Dependent(y) & Independent(x) & & & \\
\hline Rt stapes Weight & Rt Height & 0.29 & 0.26 & $y=1.7641+1.5866 x$ \\
\hline Lt Stapes Weight & Lt Height & 0.20 & $<0.0001$ & $\mathrm{y}=2.4625+0.2467 \mathrm{x}$ \\
\hline Rt Stapes Weight & Rt Length of foot plate & 0.89 & 0.61 & $\mathrm{y}=0.1089+1.0662 \mathrm{x}$ \\
\hline Lt Stapes Weight & Lt Length of foot plate & 0.09 & $<0.0001$ & $\mathrm{y}=2.8722+0.1351 \mathrm{x}$ \\
\hline Rt stapes Weight & Rt Breath of foot plate & 0.06 & 0.07 & $\mathrm{y}=1.0767+2.2458 \mathrm{x}$ \\
\hline Lt Stapes Weight & Lt Breath of foot plate & 0.89 & 0.20 & $y=0.2501+2.5014 x$ \\
\hline
\end{tabular}

\section{Discussion:-}

Current study has evaluated various dimensions of ossicles and were compared with previous results in table no. $7(2,3,4,5,6,7,8,9,10), 8(3,11,12,4,3,5,6,14,7,8,9,10)$ and $9(3,15,4,9)$. Apart from these dimensions the present study has also evaluated a positive correlation between the dimensions and weight of the ossicles which may be useful in the designing of the prosthesis. The distance between the facial nerve and ossicles was also evaluated which is an important structure to be preserved in ossiculoplasty and also its branch chorda tympani course was seen which in $80 \%$ of the observed cases traversed medial to the manubrium. Also weight of incus is more than malleus in our finding and minimum that of stapes. Incus was stouter than malleus. 
Table no 7:- Comparative morphometric data of middle ear ossicles of present study with previous studies.

\begin{tabular}{|c|c|c|c|c|c|c|c|c|c|c|}
\hline & Previous & studies & & & & & & & & \\
\hline Parameters & $\begin{array}{l}\text { Vinay } \\
\text { Chand } \\
\text { er et al } \\
2014\end{array}$ & $\begin{array}{l}\text { Padmi } \\
\text { ni et al } \\
2014\end{array}$ & $\begin{array}{l}\text { Erdogna } \\
n \text { et al. } \\
2002\end{array}$ & $\begin{array}{l}\text { Ayca } \\
\text { n et } \\
\text { al. } \\
1990\end{array}$ & $\begin{array}{l}\text { Arensbe } \\
\text { rg Et al, } \\
1981\end{array}$ & $\begin{array}{l}\text { Harada(19 } \\
72\end{array}$ & $\begin{array}{l}\text { Arensberg } \\
\text { \&nathan, } 19 \\
71\end{array}$ & $\begin{array}{l}\text { Bouch } \\
\text { et \& } \\
\text { Giraut } \\
1969\end{array}$ & $\begin{array}{l}\text { Masa } \\
\text { li } \\
1968\end{array}$ & $\begin{array}{l}\text { Prese } \\
\text { nt } \\
\text { study }\end{array}$ \\
\hline $\begin{array}{l}\text { Total } \\
\text { length(a-b) }\end{array}$ & 7.45 & 5.54 & 7.7 & 8.1 & 7.8 & 8 & 7.3 & 7.9 & 7.6 & 7.95 \\
\hline $\begin{array}{l}\text { Length of } \\
\text { manubrium( } \\
\text { b-c) }\end{array}$ & - & 3.03 & 4.7 & 4.9 & 4.4 & 4.2 & 3.5 & 4.7 & 4.6 & 4.65 \\
\hline $\begin{array}{l}\text { Length of } \\
\text { head and } \\
\text { neck }(\mathrm{a}-\mathrm{c})\end{array}$ & - & 2.79 & 4.9 & 5.1 & - & 5 & - & - & - & 2.55 \\
\hline $\begin{array}{l}\text { Weight } \\
(\mathrm{mg})\end{array}$ & 18.25 & - & - & - & - & - & - & - & - & 23.5 \\
\hline $\begin{array}{l}\text { Diameter of } \\
\text { head }(d)\end{array}$ & - & -- & - & - & - & - & - & - & - & 2.44 \\
\hline
\end{tabular}

Metric values of malleus given in $\mathrm{mm}$ and $\mathrm{mg}$

Table no 8:- Comparative morphometric data of middle ear ossicles of present study with previous studies.

\begin{tabular}{|c|c|c|c|c|c|c|c|c|c|c|c|c|c|}
\hline $\begin{array}{l}\text { Parame } \\
\text { ters }\end{array}$ & $\begin{array}{l}\text { Pad } \\
\text { mini } \\
\text { et al } \\
2014\end{array}$ & $\begin{array}{l}\text { Ka } \\
\text { mal } \\
\text { et al } \\
201 \\
0\end{array}$ & $\begin{array}{l}\text { Nate } \\
\text { kar } \\
\& \\
\text { De } \\
\text { Souz } \\
\text { a } \\
2006\end{array}$ & $\begin{array}{l}\text { Erdog } \\
\text { nan et } \\
\text { al. } \\
2002\end{array}$ & $\begin{array}{l}\text { Unu } \\
\text { r, } \\
\text { Ulg } \\
\text { er, } \\
\text { Eki } \\
\text { nci } \\
\text { (20 } \\
02\end{array}$ & $\begin{array}{l}\text { Ayc } \\
\text { an } \\
\text { et } \\
\text { al. } \\
199 \\
0\end{array}$ & $\begin{array}{l}\text { Arens } \\
\text { berg } \\
\text { Et al, } \\
1981\end{array}$ & $\begin{array}{l}\text { Harnej } \\
\text { a \& } \\
\text { Chatur } \\
\text { vedi } \\
1973\end{array}$ & $\begin{array}{l}\text { Harada( } \\
1972\end{array}$ & $\begin{array}{l}\text { Arensber } \\
\text { g } \\
\text { \&nathan, } \\
1971\end{array}$ & $\begin{array}{l}\text { Bouc } \\
\text { het } \\
\& \\
\text { Gira } \\
\text { ut } \\
1969\end{array}$ & $\begin{array}{l}\text { Mas } \\
\text { ali } \\
196 \\
8\end{array}$ & $\begin{array}{l}\text { prese } \\
\text { nt } \\
\text { stud } \\
\mathrm{y}\end{array}$ \\
\hline $\begin{array}{l}\text { Total } \\
\text { length } \\
\text { of long } \\
\text { process } \\
\text { (d-e) }\end{array}$ & 5.13 & $\begin{array}{l}6.67 \\
4\end{array}$ & 6.52 & 6.5 & 6.5 & 6.7 & 6.8 & & 6.4 & 6.4 & 6.5 & 6.4 & $\begin{array}{l}6.54 \\
5\end{array}$ \\
\hline $\begin{array}{l}\text { Distanc } \\
\text { e } \\
\text { betwee } \\
n \text { the } \\
\text { pocesse } \\
\mathrm{s}(\mathrm{ef})\end{array}$ & 4.5 & 6.01 & 5.86 & 6.1 & 6.1 & 6.1 & - & - & 4.2 & - & - & - & 5.97 \\
\hline $\begin{array}{l}\text { Total } \\
\text { length } \\
\text { of short } \\
\text { process } \\
\text { (d-f) }\end{array}$ & 3.47 & $\begin{array}{l}5.04 \\
3\end{array}$ & 5.06 & 4.9 & 4.9 & 5.1 & 5.1 & - & 4.8 & 5.1 & 5.1 & 4.8 & $\begin{array}{l}5.46 \\
5\end{array}$ \\
\hline $\begin{array}{l}\text { Weight } \\
\text { (mg) }\end{array}$ & - & $\begin{array}{l}26.3 \\
03\end{array}$ & $\begin{array}{l}20.7 \\
4\end{array}$ & - & - & - & - & 25.06 & - & - & - & - & $\begin{array}{l}26.7 \\
8\end{array}$ \\
\hline Angle & - & $\begin{array}{l}74.3 \\
42 \\
\end{array}$ & - & - & - & - & - & - & - & - & - & - & $\begin{array}{l}106 . \\
335\end{array}$ \\
\hline
\end{tabular}

Metric values of incus given in $\mathrm{mm}$ and $\mathrm{mg}$. 
Table no 9:- Comparative morphometric data of middle ear ossicles of present study with previous studies.

\begin{tabular}{|c|c|c|c|c|c|}
\hline & \multicolumn{4}{|l|}{ Previous study } & \multirow[b]{2}{*}{ present study } \\
\hline Parameters & $\begin{array}{l}\text { Padmini et al } \\
2014\end{array}$ & $\begin{array}{l}\text { Wadhwa et al } \\
2005\end{array}$ & $\begin{array}{l}\text { Erdognan et al. } \\
2002\end{array}$ & $\begin{array}{l}\text { Bouchet \& } \\
\text { Giraut } 1969\end{array}$ & \\
\hline Total height $(\mathrm{gh})(\mathrm{mm})$ & 2.71 & 3.4 & 3.2 & 3.5 & 3.26 \\
\hline length of footplate(ij)(mm) & 2.36 & 2.97 & 2.6 & & 3.02 \\
\hline $\begin{array}{l}\text { breadth of foot plate } \\
(\mathrm{kl})(\mathrm{mm})\end{array}$ & 1.91 & & 1.3 & & 1.19 \\
\hline weight (mg) & & & & & 3.275 \\
\hline
\end{tabular}

Metric values of stapes given in $\mathrm{mm}$ and $\mathrm{mg}$.

\section{Conclusion:-}

Osteometric dimensions are useful in prosthesis designing which can be used in ossicular reconstruction. Also the ear ossicles may be used to create a bank for allograft in ossiculoplasty.

Consent -not applicable

Ethical approval - not applicable as study is on cadavers in department of anatomy

Acknowledgement - We would like to thank PHOD Anatomy GMC Kota and PHOD Pathology GMC Kota.

Interest of conflict- none

\section{References:-}

1. Earnest Albert Hooton:Up from the ape page 63-64 $1^{\text {st }}$ edition: The macmillian company new York 1946.

2. Vinaychandra, P.H.,Viveka S, Sudha M J, Balakrishna Shetty, Santhosh Kurikose, Srinivasa Sagar: Morphometry and variations of malleus with clinical correlations: International Journal of anatomy an research 2014:2(1):191-94

3. Pramila Padmini and B. Narasinga Rao: Morphometry of Human Fetal Ear Ossicles: A Human Cadaveric Study: M.:British Journal of Medicine \& Medical Research 4(9): 1873-1882, 2014

4. Erdoğan Unur, Harun Ülger, Nihat Ekinci, Erciyes Tip Dergisi Morphometrical And Morphological Variations Of Middle Ear Ossicles In The Newborn (Erciyes MedicalJournal). 2002;24(2):57-63.

5. Aycan K, Unur E, Bozkır MG, et al. Anatomical study of malleus. Journal of Health Sciences. 1990;1:152-158.

6. Arensburg B, Harell M, Nathan H (1981) The Human Middle Ear Ossicles: Morphometry and taxonomic implications. J Hum Evol 10: 199-205.

7. Harada O, Ishii H. The Condition of the auditory ossicles in microtia. Plast Reconst Surg. 1972;50:48-53

8. Arensburg B, Nathan H. Observations on a notch in the short (Superior or Posterior) process of the incus. Acta Anat. 1971;78: 84-90.

9. Bouchet A, Giraud M (1968) Contribution a L'etude morphologique et radiologique des osselets de L'ouie. Compte rendu de L' Association des Anatomists 53 Congress 141: 588-600.

10. Masali M., 1968. The ear bones and the vertebral column as indicators of taxonomic and postural distinction among Primates. In B. Chiarelli (ed.)Taxonomy and Phylogeny of the Old World Primates with reference to the Origin of Man. Rosemberg and Sellier, Torino. pp. 69-94.

11. Kamal Singh, Aarti Rohilla and Jyoti Rohila: Incus Morphometry: A Possible Tool in Sex Determination,J Forensic Res 2016, 7:2

12. Natekar PE, De Souza FM (2006) A morphometric study of malleus and incus and its clinical implications. Indian J Otol 12: 6-9.

13. Erdoğan Unur, Harun Ülger, Nihat Ekinci, Erciyes Tip Dergisi Morphometrical And Morphological Variations Of Middle Ear Ossicles In The Newborn (Erciyes MedicalJournal). 2002;24(2):57-63

14. Harneja NK, Chaturvedi RP (1973) A Study of the Human Ear Ossicles. Indian J Otol 25: 154-160

15. Wadhwa,S., Kaul,J.M. and Agarwal, A.K.: Morphometry study of stapes and its clinical implications.Journal of Anatomical Society of India 54(2)1-9(2005). 